\title{
An improved magnetic model for thermodynamic modeling
}

\author{
Wei Xiong $^{a^{*}}$, Qing Chen $^{b}$, Pavel Korzhavyi $^{a}$, Malin Selleby $^{a}$ \\ a Department of Materials Science and Engineering, KTH Royal Institute of Technology, \\ SE-100 44 Stockholm, Sweden \\ b Thermo-Calc software AB, Norra Stationsgatan 93, SE-113 64 Stockholm, Sweden \\ * Corresponding author: wxiong@yahoo.com, Tel.: +46-8 790 8313, Fax: +46-820 \\ 7681
}

\begin{abstract}
The standard magnetic model in the current CALPHAD modeling is based on the IndenHillert-Jarl model and an empirical constraint due to Weiss and Tauer that can be used to connect ferromagnetism and antiferromagnetism. In this work, we demonstrate that many artifacts can be produced by using the current approach when modeling systems with elements of different forms of magnetism. We then propose several simple measures to improve the standard magnetic model so that a physically and numerically correct and more accurate description for the Gibbs energy of magnetic ordering can be obtained in normal situations. Especially, we have assumed that each magnetic phase always possesses both ferromagnetic and antiferromagnetic states, with one of them stable and the other non-stable. The concept of 'effective magnetic moment' has also been introduced as a measure of the maximum magnetic entropy. A case study on the Al-Cr-Fe system has been performed at $0 \mathrm{~K}$ showing the importance of reasonable description of the magnetic phase diagrams. It has been stressed that the extended magnetic model in this work can be further employed for bridging atomistic and phenomenological
\end{abstract}


modeling for multi-scale simulation.

Keywords: magnetic ordering; thermodynamics; phase diagram; ab initio; Al-Cr-Fe 


\section{Introduction}

Magnetism is a physical phenomenon of fundamental importance in many applications. Recent years have witnessed unprecedented interest in the study of magnetic phenomena and their effects by both physicists and material scientists [1-6]. The impact of magnetism upon metallurgy was firstly discussed by Zener in 1955 [7]. The significance of evaluating the magnetic contribution to the Gibbs energy was strengthened in Zener's work by proposing a simple model for alloy theory, in which the free energy curve for pure iron was rigidly shifted in order to represent the alloying effects on the Curie temperature. At the same time, Tauer and Weiss [8] developed a method to evaluate the specific heats of ferromagnetic (FM) and antiferromagnetic (AFM) states, by separating the magnetic contribution from vibrational and electronic contributions. Following the idea of Tauer and Weiss [8], a magnetic model for thermodynamic modeling was proposed by Inden [9] in 1976, and later revised slightly by Hillert and Jarl [10] in order for the model to be easily implemented in computer programs, such as Thermo-Calc, for thermodynamic calculations. In 1982, Hertzman and Sundman [11] applied the model to describe the $\mathrm{Fe}-\mathrm{Cr}$ system where $\mathrm{Fe}$ and $\mathrm{Cr}$ exhibit different forms of magnetism, i.e. ferromagnetism and antiferromagnetism, respectively in the bcc structure. They followed Weiss and Tauer's proposal [12] and scaled the Néel temperatures and magnetic moments with an AFM factor, which is -3 for the fcc phase and -1 for the bcc phase. In this way, composition dependences of both the Curie and Néel temperatures over the whole composition range could be described by using only one smooth curve and the transition from FM to AFM ordering occurs when the critical temperature curve passes through zero. For convenience, the same factor has also been used for the composition 
dependence of the mean magnetic moment. Although there are some other suggestions [13] based on Inden's idea, the one given by Hillert and Jarl [10] for FM ordering transition and its further extension by introducing an antiferromagnetism factor for AFM ordering transition [12] are generally accepted as the standard magnetic model in the CALPHAD (CALculation of PHAse Diagrams) community and have been adopted in all available software packages. This model has been widely used for evaluating the magnetic ordering energy in thermodynamic models, and also for predicting the magnetic influence on diffusivities used in kinetic simulations.

The importance of reasonably describing the magnetic ordering energy and its influence on diffusion cannot be underestimated. For example, in the work by Xiong et al. [14], it was demonstrated the model-predicted $T_{0}$ curve (the temperatures curve showing the same value of the Gibbs energy for both fcc and bcc phases) in the previous thermodynamic descriptions can be improved significantly by just improving the description of the magnetic phase diagram in the Fe-Ni system. As to the impact on diffusion-controlled phase transformation kinetics, the diffusivity of components in the magnetic system will be affected significantly at low temperatures where magnetism plays a critical role because the magnetic enthalpy alters the activation energy for diffusion. When coupling the phase field modeling with CALPHAD thermodynamics and kinetics, as discussed in the work by Xiong et al. [15] using the case of Fe-Cr, inadequate description of magnetic ordering energy makes it impossible to accurately simulate microstructural evolution in magnetic materials on a real time scale.

One of the major advantages of the CALPHAD approach is its applicability to multicomponent systems. Numerous computational thermodynamic studies have been 
performed in the last three decades, but unfortunately not much attention has been paid to the magnetic phase diagrams in the thermodynamic modeling of magnetically ordering systems. As a result, considerable amount of CALPHAD-type descriptions for magnetic phase diagrams are rudimentary. This is probably due to three factors. Firstly, most magnetic transitions occur at low temperatures, which were beyond the interest of CALPHAD in the early years. The lack of accurate thermodynamic description below $300 \mathrm{~K}$ has generated a gulf between atomistic modeling, e.g. ab initio studies and CALPHAD modeling. A good reflection of this is the absence of lattice stability below $300 \mathrm{~K}$. Secondly, due to the complexity of magnetism, a comprehensive review on the measurement of magnetic properties sometimes calls for more efforts than merely evaluating the thermodynamic properties for the system under consideration. Thirdly, the ultimate goal of thermodynamic modeling is normally about phase equilibria, phase diagrams, and thermodynamic properties, which are mostly measured without any link to the measurement of magnetic properties.

Recently, in the work on the Fe-Cr and Fe-Ni systems [14, 16, 17], it was found that the magnetic phase diagrams have been poorly evaluated even for these fundamental binaries with extreme importance in practical applications. Moreover, it has been reported in the work by Xiong et al. [14] that a difficulty arises when trying to describe the magnetic transition temperature for both antiferromagnetism and ferromagnetism of the fcc phase in the Fe-Ni system with only one Redlich-Kister (R-K) polynomial [18] assisted by the AFM factor. In order to avoid such a difficulty, and have a remedy for the situation, we intend in this work to modify the standard magnetic model that is currently used in the CALPHAD community and establish a more accurate description of the 
magnetic ordering energy in multicomponent systems exhibiting several different forms of magnetism.

The IHJ model and the introduction of the AFM factor in the CALPHAD approach will be firstly described in Section 2 in order to compare with the improvements made in this work shown in Section 3. The various issues and remaining problems will be further discussed in Section 4 and 5.

\section{The IHJ model and the AFM factor}

The IHJ model $[9,10]$ has been available since 1978, and the original work only discussed the transition in FM states but not AFM states. The IHJ model was later adopted in the thermodynamic evaluation of the Fe-Cr system by Hertzman and Sundman [11], who also introduced the AFM factor suggested by Weiss and Tauer [12] in order to describe the AFM ordering transitions. In this section, we will firstly elucidate the way to apply the IHJ model in the current CALPHAD method, and then make some necessary comments.

\subsection{The original IHJ model}

The magnetic model proposed by Inden [9] is based on two practical ideas. Firstly, the magnetic contribution to heat capacity $\left(\mathrm{C}_{\mathrm{P}}\right)$ was expressed in an analytic form as follows:

$$
\left\{\begin{array}{l}
C_{P}^{L R O}=K^{L R O} \cdot R \cdot \ln \frac{1+\tau^{3}}{1-\tau^{3}}, \quad \tau<1 \\
C_{P}^{S R O}=K^{S R O} \cdot R \cdot \ln \frac{1+\tau^{-5}}{1-\tau^{-5}}, \quad \tau>1
\end{array}\right.
$$

where $\tau$ is $T / T_{C}, T_{C}$ the Curie temperature, $C_{P}^{L R O}$ and $C_{P}^{S R O}$ correspond to the magnetic contribution to the heat capacity in the FM and paramagnetic (PM) states. $K^{L R O}$ and 
$K^{S R O}$ are two constants for FM and PM states. $R$ is the gas constant.

Secondly, based on the work of Tauer and Weiss [8], Inden suggested to calculate the maximum magnetic entropy of an element undergoing the FM disordering transition using a function of mean magnetic moment $(\bar{\beta})[9]$ :

$$
S_{\max }^{\operatorname{magn}}=R \cdot \ln (\bar{\beta}+1)
$$

Based on the above ideas, Hillert and Jarl [10] found that it is sufficient to use a truncated Taylor expansion form of Eq. (1):

$$
\begin{cases}C_{P}^{L R O}=2 K^{L R O} \cdot R \cdot\left(\tau^{m}+\frac{1}{3} \tau^{3 m}+\frac{1}{5} \tau^{5 m}\right), & \\ C_{P}^{S R O}=2 K^{S R O} \cdot R \cdot\left(\tau^{-n}+\frac{1}{3} \tau^{-3 n}+\frac{1}{5} \tau^{-5 n}\right), & \tau>1\end{cases}
$$

where $m=3$ and $n=5$. Therefore, the final expression of the Gibbs energy for magnetic ordering becomes:

$$
\begin{aligned}
& G_{m}^{m a g n}=R T \cdot \ln (\bar{\beta}+1) \cdot g(\tau) \\
& g(\tau)= \begin{cases}1-\frac{1}{A}\left[\frac{79 \tau^{-1}}{140 p}+\frac{474}{497}\left(\frac{1}{p}-1\right)\left(\frac{\tau^{3}}{6}+\frac{\tau^{9}}{135}+\frac{\tau^{15}}{600}\right)\right], & \tau<1 \\
-\frac{1}{A}\left(\frac{1}{10} \tau^{-5}+\frac{1}{315} \tau^{-15}+\frac{1}{1500} \tau^{-25}\right), & \tau \geq 1\end{cases} \\
& A=\frac{518}{1125}+\frac{11692}{15975}\left(\frac{1}{p}-1\right)
\end{aligned}
$$

where $p$ is called the structure factor, which is the ratio of magnetic enthalpy in the PM state to the total magnetic enthalpy. For the bcc structure, the accepted value is: $p=0.4$, while for fcc and hcp crystals, $p=0.28$ [9]. Normally, $p$ can be used to determine the 
shape of the $\lambda$-peak in the $\mathrm{Cp}$ curve due to the magnetic transition.

One can readily derive the above expression for the Gibbs energy of magnetic ordering using a basic thermodynamic relation:

$$
G^{\text {magn }}= \begin{cases}\int_{T_{1}}^{T} C_{P}^{L R O} d T-T \int_{T_{1}}^{T} \frac{C_{P}^{L R O}}{T} d T+\int_{\infty}^{T_{1}} C_{P}^{S R O}\left(1-\frac{T_{1}}{T}\right) d T & \left(T \leq T_{1}\right) \\ \int_{\infty}^{T} C_{P}^{S R O} d T-T \int_{\infty}^{T} \frac{C_{P}^{S R O}}{T} d T & \left(T>T_{1}\right)\end{cases}
$$

where $T_{1}$ is the magnetic transition temperature for integration. It is noteworthy that the fully disordered magnetic state, i.e. the ideal paramagnetic state, at the infinitely high temperature has been chosen as the reference state in this expression. As explained in the work of Inden [19], an artificial miscibility gap could occur in some magnetic systems showing strong magnetism but containing components showing weak or even no magnetic ordering, e.g. in the bcc $\mathrm{Fe}-\mathrm{Al}$ case, if the ordered magnetic state at low temperature is considered as the reference state.

\subsection{Mean magnetic moment and local magnetic moment}

An important parameter in the above model is the local magnetic moment of the atoms $(\beta)$, which determines the value of the maximum magnetic entropy and controls the intensity of the $\lambda$-peak in $C_{P}$ curve at the magnetic transition temperature. It has been suggested by Inden [9] the expression using the mean magnetic moment for the maximum magnetic entropy in Eq.(2) approximates the more general expression for the FM ordering:

$$
S_{\max }^{\text {magn }}=R \sum_{i} x_{i} \ln \left(\beta_{i}+1\right)
$$

which should be used if the local magnetic moments of the component atoms $i$ are known. 
Apparently, the difference between the exact and approximate value of $S_{\max }^{\operatorname{magn}}$ depends on their inputs, i.e. the local magnetic moments and the mean magnetic moment. We can get a sense of the difference by taking an example, the bcc Fe-Ni system from a previous work by Xiong et al. [14]. As shown in Fig. 1(a), the difference between exact maximum magnetic entropy and approximate one shows a maximum at c.a. 50 at.\% Ni.

It is worth mentioning that both local magnetic moment and mean magnetic moment can be determined by using neutron magnetic scattering and some other techniques [20]. Moreover, mean magnetic moment for FM materials can also be determined via measurement of saturation magnetization [14, 21]. Despite this, in current CALPHAD modeling, only mean magnetic moment is used as the input, even though in some cases the local magnetic moment can be well determined via experiments or ab initio calculations $[14,22-24]$.

In this work, a thermodynamically effective magnetic moment is introduced and suggested to be used in CALPHAD modeling if local magnetic moment is available. In short, it is called "effective magnetic moment" and denoted $\beta^{*}$, which satisfies the following relation.

$$
S_{\max }^{\operatorname{magn}}=R \cdot \ln \left(\beta^{*}+1\right)=R \cdot \sum_{i} x_{i} \cdot \ln \left(\beta_{i}+1\right)
$$

It is obvious that the effective magnetic moment $\beta^{*}$ can be calculated from the local magnetic moments:

$$
\beta^{*}=\prod_{i}\left(\beta_{i}+1\right)^{x_{i}}-1
$$

The obtained effective magnetic moment can then be described by using a single R-K polynomial. The differences between $\beta^{*}$ and $\bar{\beta}$ for the bcc Fe-Ni alloys are visible in Fig. 
1(b). It should be emphasized that the effective magnetic moment $\beta^{*}$ and local magnetic moments $\beta_{i}$ will generate the same value for the maximum magnetic entropy, while $\bar{\beta}$ will normally give a different value as an approximation.

\subsection{The AFM factor and possible resulted artifacts}

It should be noted that the IHJ model was introduced to describe only the FM ordering in the work by Inden [9] and Hillert and Jarl [10]. None of them discussed the AFM state or a system with composition regions of different magnetic states. The task was left to Hertzman and Sundman [11] when they investigated the Fe-Cr system.

Hertzman and Sundman [11] have successfully applied the IHJ model for both FM and AFM states in the $\mathrm{Fe}-\mathrm{Cr}$ system, where $\mathrm{Fe}$ and $\mathrm{Cr}$ exhibit ferromagnetism and antiferromagnetism, respectively, in the bcc structure. In order to have a single expression for the composition dependences of both Curie and Néel temperatures, they adopted Weiss and Tauer's proposal [12] that the parameters for AFM ordering can be treated as negative values divided by a factor, which is -3 for the fcc structure and -1 for the bcc structure. The whole scheme is illustrated in Fig. 2(a) and (b). It was based on Weiss and Tauer's observation on quite a number of binary systems and worked well for Hertzman and Sundman's purpose [11]. It should be mentioned that the same AFM factors have also been adopted by them for describing the mean magnetic moment in the Fe-Cr system. Ever since then, this treatment has become an important part of the standard magnetic model used in the CALPHAD community and has been generalized without any further examination and questioning for all different kinds of systems.

Now let's have a look of a case where an element exhibiting no magnetic ordering is involved, see Fig. 2(c) and (d), we found immediately that an artificial Néel temperature 
curve is resulted on the side of component A showing weak magnetic behavior. It is easy to envisage that if the composition at which the type of magnetism changes at $0 \mathrm{~K}$ in Fig. 2(c) or (d) is closer to the pure component showing FM ordering, the artificial Néel temperatures at the side of component A will be more pronounced, and will thus result in significant artificial AFM contributions to the Gibbs energy in this artificial AFM composition range. A second case is shown in Fig. 3(a) to demonstrate that it is not viable to use a single R-K polynomial to describe different magnetic regions. A practical study for this problem is discussed in the work on the Fe-Ni system by Xiong et al. [14]. In that case, the Néel temperature curve for the fcc phase close to pure Fe will have a maxima. In addition, the composition at which the form of magnetism changes at $0 \mathrm{~K}$ could not be fitted to 25 at.\% Ni, which has been predicted by ab initio calculations [25]. Forcing this composition will lead to an artificial maximum Néel temperature of more than $298 \mathrm{~K}$ on the Fe-rich side. The situation may be even worse if the change from AFM to FM at $0 \mathrm{~K}$ is not continuous as shown in Fig. 3(b). In this case, there is no chance to be able to model the PM region at $0 \mathrm{~K}$ with a single critical temperature curve by using the AFM factor.

As we mentioned before, the mean magnetic moment has been used as an input parameter of the IHJ model in the current CALPHAD modeling and its composition dependence was described in a similar way as that for the critical temperature with the same AFM factor for an alloy phase of different types of magnetism in different composition regions. This approach could lead to a conceptual error, which can be demonstrated by using Fig. 2(c) and (d) as an example and considering the y-axis as $\bar{\beta}$, instead of magnetic transition temperature. In this case, undesired non-zero mean 
magnetic moments will be obtained for alloys in the PM region with vanished global magnetization.

\section{A revised magnetic model}

In order to eliminate the artifacts revealed above and describe the magnetic Gibbs energy more accurately, herewith we propose some modifications in the standard magnetic model used in the current CALPHAD modeling

\subsection{Effective magnetic moment}

We have suggested in the above section that, although the difference between using the effective and mean magnetic moment may not be so large in many cases with FM ordering transition, the former one should be preferred if experimental information on the local magnetic moments is available. In order to represent the maximum magnetic entropy more exactly, one should always consider the effective magnetic moment rather than the mean magnetic moment if possible. It is worth mentioning that the effective magnetic moment is a representation of the local magnetic moment, which can sometimes be readily calculated by using the ab initio method. Therefore, the ab initio calculations become an important tool for supplying information on the effective magnetic moment if the experimental data is not available.

By using the effective magnetic moment as input to the IHJ model, we can not only obtain more exact maximum magnetic entropy, but also avoid the conceptual error we found with the standard approach in the above section. Now we shall illustrate our solution by using a hypothetical system shown in Fig. 4 with an AFM-FM transition at 0 $\mathrm{K}$, which is an archetype for the fcc Fe-Ni alloy [14]. By using the approach in the current CALPHAD modeling, the mean magnetic moment will be modeled as the short- 
dashed line (see Fig. 4(b)). Besides, in order to describe the mean magnetic moment using a single R-K polynomial with the help of the AFM factor, a line shown as a dashed curve, i.e. non-zero values will appear in the AFM region, which is physically incorrect since the global magnetization in an ideal AFM state will vanish. However, if the local magnetic moments are available, one could instead use the thermodynamically effective magnetic moment which will give the solid line instead in Fig. 4(b).

It is noteworthy that the composition at which the mean magnetic moment curve pass through $0 \mathrm{~K}$ could be used to identify the FM/AFM or FM/PM transition point at $0 \mathrm{~K}$, and the point should have the same composition as the one for the magnetic transition temperature curve at $0 \mathrm{~K}$. This may be useful when experimentally low magnetic transition temperature is hard to determine and the experimental mean magnetic moment is not available. In this situation, one could try to compute the mean magnetic moment by using ab initio calculations and identify the critical composition where the type of magnetism changes at ground state.

In fact, the effective magnetic moment in the present magnetic model should be ultimately served for accurately evaluating the magnetic ordering energy. In the CALPHAD-type description of pure elements, the effective magnetic moment may needs to adjust in a way to reproduce the experimental heat capacity satisfactorily. For instance, in the study of bec Cr by Andersson [26], an artificial value of $0.008 \mu_{\mathrm{B}}$ is adopted for the magnetic moment, which was called thermodynamically magnetic moment, since the magnetic moment cannot be defined precisely in the case of $\mathrm{Cr}$ with spin density wave effects, and the measured value for magnetic moment will generate more pronounced peak in the $\mathrm{Cp}$ curve. Another interesting example can be found in fcc Ni. The 
experimental study shows a value of $0.62 \mu_{\mathrm{B}}$ for the magnetic moment of pure $\mathrm{Ni}$, but the adopted value in the CALPHAD database is $0.52 \mu_{\mathrm{B}}$ [27], which is also the result of fitting the $\lambda$-shape peak of the $\mathrm{Cp}$ curve precisely according to the experimental data.

\subsection{Different magnetic states of a phase}

We propose to introduce both FM and AFM states for each phase exhibiting FM or AFM in reality, which means that the hidden state is non-stable. For each magnetic state, no matter if it is stable or non-stable, there exists a corresponding magnetic transition temperature. Furthermore, we assume that for each phase there is only one stable magnetic state. Therefore, a negative absolute magnetic transition temperature is assigned for each phase in its non-stable magnetic state. For convenience, we take the absolute value of this magnetic transition temperature the same as that for the stable magnetic state. In this way, the composition dependences of the Curie and Néel temperatures will be described separately using two different R-K polynomials, and the empirical AFM factor applied in the standard magnetic model currently used in CALPHAD community is not necessary. As a result, the artifact caused by using one single polynomial with help of the AFM factor will disappear.

Some archetypes of magnetic phase diagrams are illustrated in Fig. 5 by showing the way of fitting the magnetic transition temperature using the revised model proposed in this work.

\subsection{Gibbs energy of magnetic ordering}

It is natural now to set the magnetic contribution to the total Gibbs energy to zero once the magnetic critical temperature becomes negative. It should be noticed that in an 
earlier work by Chen and Sundman [28] some modifications of the expression for the magnetic heat capacity was done in order to reproduce more exactly the well-determined experimental enthalpy of the phase transformation between bcc and fcc for pure Fe. The polynomial shown in Eq. (3) is further added with one more expansion term as shown below.

$$
\begin{cases}C_{P}^{L R O}=2 K^{L R O} \cdot R \cdot\left(\tau^{m}+\frac{1}{3} \tau^{3 m}+\frac{1}{5} \tau^{5 m}+\frac{1}{7} \tau^{7 m}\right), & \\ C_{P}^{S R O}=2 K^{S R O} \cdot R \cdot\left(\tau^{-n}+\frac{1}{3} \tau^{-3 n}+\frac{1}{5} \tau^{-5 n}+\frac{1}{7} \tau^{-7 n}\right), & \tau>1\end{cases}
$$

The power exponent, $n$, shown in the above equation was adjusted to 7, while $m$ is kept to 3. Therefore, $K^{L R O}$ and $K^{S R O}$ have been re-optimized and yields new values for the structural factor, $p=0.37$ for the bcc crystal, and $p=0.25$ for the non-bcc phases. It has been showed that the slight change of the $p$ value will not bring any strong effects to the fitting of heat capacity for pure Co and $\mathrm{Ni}$ [28].

Accordingly, in this work, the basic function of the Gibbs energy of magnetic ordering has been adopted as:

$$
\begin{gathered}
G_{m}^{\text {magn }}=R T \ln \left(\beta^{*}+1\right) \cdot g(\tau) \\
g(\tau)=\left\{\begin{array}{l}
0, \tau \leq 0 \\
1-\frac{1}{D}\left[0.38438376 \frac{\tau^{-1}}{p}+0.63570895\left(\frac{1}{p}-1\right)\left(\frac{\tau^{3}}{6}+\frac{\tau^{9}}{135}+\frac{\tau^{15}}{600}+\frac{\tau^{21}}{1617}\right)\right], 0<\tau \leq 1 \\
-\frac{1}{D}\left(\frac{1}{21} \tau^{-7}+\frac{1}{630} \tau^{-21}+\frac{1}{2975} \tau^{-35}+\frac{1}{8232} \tau^{-49}\right), \quad \tau>1 \\
D=0.33471979+0.49649686\left(\frac{1}{p}-1\right)
\end{array}\right.
\end{gathered}
$$

It should be emphasized that the reference state of the magnetic ordering effects is the 
disordered state at the infinitely high temperature. As a consequence, at $0 \mathrm{~K}$ the magnetic entropy does not go to zero, but $-R \ln \left(\beta^{*}+1\right)$, i.e., minus the absolute value of the maximum magnetic entropy. Therefore, in order to enforce the third law of thermodynamics for the magnetic state at $0 \mathrm{~K}$, the nonmagnetic entropy is shifted by $R \ln \left(\beta^{*}+1\right)$ in order to compensate for the magnetic entropy at $0 \mathrm{~K}$.

\section{Application of the improved model in multicomponent systems}

The improved magnetic model can be further applied to multicomponent systems. Sometimes one may need to concern the extrapolation into the ternary phase region as the case demonstrated in Fig. 6. Comparing with the standard model currently used in the CALPHAD community, the improved model outlined above is able to describe more cases regarding ferromagnetism, antiferromagnetism and non-magnetism without producing any artificial magnetic contributions to the total energy. According to the illustration in Fig. 6, the crossover between the Curie and Néel temperature curves should be lower than $0 \mathrm{~K}$, which means there is no region for ferromagnetism and antiferromagnetism coexisting above $0 \mathrm{~K}$. It should be noted that in the magnetic phase diagram, like the A-B-D system, shown in Fig. 6, the fitting for the effective magnetic moment may show some parts with negative values (see the corner of component B in Fig. 6(b)). It is thus assumed that such a range will have no contribution to the magnetic entropy.

A case study on the bcc phase of the Al-Cr-Fe magnetic phase diagram is performed in this work and its results are presented in Figs. 7-9. Firstly, according to the experimental data [29-39], the magnetic phase diagram has been constructed by plotting the magnetic transition temperatures and magnetic moments as shown in Figs. 7 and 8, respectively. It 
should be noted that the ternary region is the simple extrapolation from the binaries, without introducing any ternary interaction parameters, due to lack of experimental data. From Fig. 7, it follows that none of the magnetic temperature curves (see red dashed lines) have been described correctly in the reported assessments [24, 40, 41]. By using the improved magnetic model, the binaries, Al-Fe and Al-Cr, can be described satisfactorily without generating any artificial Néel temperatures at the non-magnetic sides (see blue lines). It is easy to envision that the magnetic contributions calculated with the model in this work will be significantly different from those obtained in the previous assessments $[24,40,41]$. By extrapolation from the previous descriptions of the binaries, no PM region at $0 \mathrm{~K}$ exists except for pure $\mathrm{Al}$, and $\mathrm{AFM}$ regime will dominate a much wider range compared with the one from this work.

In Fig. 9, the magnetic ordering energy at ground state has been plotted by using the standard CALPHAD magnetic model and the improved magnetic model in this work. The distribution of the magnetic ordering energy in both models shown in Fig. 9(a) and (b) are distinctly different. It is noteworthy that even though the energy difference is not large in the binary cases, the energy difference due to the description of magnetic phase diagram will be more pronounced when extrapolating into the ternary region.

\section{Discussions}

\subsection{Magnetism in pure elements}

At present, we feel the need to emphasize the importance of the description of the magnetism in pure elements, since the reliability of the description for unary will eventually affect the thermodynamic description of higher order systems. It is likely that the magnetic properties of some unary systems need to be revisited. For instance, since 
the fcc structure is unstable for pure $\mathrm{Cr}$, it may need further affirmation for adopting a Néel temperature of $369.667 \mathrm{~K}$ and a magnetic moment of $0.82 \mu_{\mathrm{B}} /$ atom as done in the SGTE database [27], which was a result by extrapolating from the other two binary Fe-Ni and $\mathrm{Cr}-\mathrm{Ni}$ systems when modeling the Fe-Cr-Co-Ni system by Chin et al. [11, 42]. Another example that is more convincing is the magnetic properties of pure Ni. As reported by Tian et al. [3], pure bcc $\mathrm{Ni}$ is experimentally stable in the FM state with a Curie temperature of $456 \mathrm{~K}$ and a magnetic moment of $0.52 \pm 0.08 \mu_{\mathrm{B}} /$ atom. These values differ quite much from the adopted values in the SGTE database $(575 \mathrm{~K}, 0.85$ $\mu_{\mathrm{B}} /$ atom).

It should be mentioned that some magnetic properties in amorphous phases are also available from experiments. A comprehensive study on the lattice stability of Fe by Chen and Sundman [28] has summarized such information for some Fe-based binaries in order to achieve a reasonable value for magnetic properties of amorphous Fe by extrapolation.

\subsection{Description of magnetism for compounds}

There are countless numbers of compounds exhibiting magnetic ordering transformations. However, in thermodynamic modeling, the magnetic transitions in compounds are often not paid enough attention. For example, in the case of $\mathrm{Fe}-\mathrm{Cr}$, the $\sigma$ phase has been determined to show ferromagnetism at c.a. $50 \mathrm{~K}[43,44]$, but this had been neglected when the system was modeled. Another issue similar to the description of many intermetallic phases is the solution phases modeled by using the sublattice model. Such cases have been demonstrated in the work of Guillermet [45] on the Co-C system, and can be further applied to the intermetallic phases. Within the compound energy formalism [46], one would need to determine the magnetic properties for end-members at first. 


\subsection{Limits of the improved magnetic model}

Due to the complexity of magnetism, one may find some phenomena which could not be adequately represented by using even the improved magnetic model proposed in this work. Specifically, for a phase with fixed composition, the present model does not allow the type of magnetism that changes forth and back as shown in Fig. 10 (the green shaded part in the vicinity of 50 at.\% B). Moreover, AFM and FM ordering are not allowed to coexist for the same structure. For example, the spinel phase $\mathrm{Co}_{3} \mathrm{O}_{4}$ has AFM at about 30 $\mathrm{K}$ and FM at about $1200 \mathrm{~K}$ [23]. Normally, as a compromise, the transition related to FM states in this case will be described, whereas the AFM states will not be considered because the transition temperature is very low, and thus the magnetic contribution to the Gibbs energy is less than FM ordering transition.

A similar intriguing case can be found in the magnetic phase diagram of the Fe- $\mathrm{Rh}$ system. A preliminary assessment has been carried out by Ohnuma et al. [47], in which the magnetic phase diagram of the ordered bcc alloys has been simplified by comparing the determined magnetic phase diagram [48] as reconstructed in Fig. 11. Certainly, the magnetic transition of the bcc alloys along the temperature axis from AFM to PM with FM in between is beyond the capability of the present magnetic model.

\subsection{Temperature and pressure dependence of magnetic transition}

It is noteworthy that the present magnetic model in the thermodynamic modeling has not included any temperature or pressure dependence of magnetization. In principle, the magnetic moments will show both temperature and pressure dependence, while the magnetic transition temperature has pressure dependence only. Further consideration in the related topics will significantly broaden the application of the present magnetic model. 
Therefore, it would be practically useful to introduce temperature dependence for the magnetic moment, which may give more reasonable description of the magnetic entropy, supposing the relation shown in Eq. (10) will still hold at higher temperatures.

\subsection{Low temperature CALPHAD}

The present work shows the importance of describing magnetic phase diagram in phase equilibria studies. It is obvious that thermodynamics at low temperatures are strongly related to magnetism in the magnetic systems. Due to considerable needs of practical applications at low temperatures of computational thermodynamic databases, nowadays, the concept of low-temperature CALPHAD is in its infancy, which emphasizes the accurate description of the low-temperature thermodynamics by integrating ab initio calculations, new generation of lattice stability [49] and experiments. These integrated methods should be considered as the tools for the inputs in the lowtemperature CALPHAD method. Apparently, the magnetic effects should be taken into account properly in the modeling at low temperatures. By improving the standard magnetic model that is currently used in CALPHAD modeling, we expect to provide a possible link to bridge different methods in the field of low-temperature CALPHAD.

\section{Summary}

In this work, we discussed problems existing in the standard magnetic model currently used in the CALPHAD community when modeling the magnetic alloy systems. It is suggested that the magnetic phase diagram should be studied carefully in order to model the thermodynamic and kinetic data correctly. It is expected that the ab initio and CALPHAD methods could be integrated by using the new generation of the lattice stability and the present magnetic model. 
This work presents an improved magnetic model for the CALPHAD method with a hope that our simple modifications may draw more attention to the description of magnetic ordering and mitigate the challenges of describing magnetic phase diagrams at the moment. Apparently, the further development of the CALPHAD method calls for more robust and sophisticated magnetic models.

\section{Acknowledgement}

This work was performed within the VINN Excellence Hero-m Center, financed by VINNOVA, the Swedish Government Agency of Innovation Systems, Swedish Industry and the Royal Institute of Technology. Authors are grateful to Profs. Emerit. Mats Hillert and Gerhard Inden for helpful discussions.

\section{References:}

[1] M. van Schilfgaarde, I.A. Abrikosov, B. Johansson, Nature (London, U. K.), 400 (1999) 46-49.

[2] A.I. Lichtenstein, M.I. Katsnelson, Phys. Rev. Lett., 87 (2001) 067205.

[3] C.S. Tian, D. Qian, D. Wu, R.H. He, Y.Z. Wu, W.X. Tang, L.F. Yin, Y.S. Shi, G.S. Dong, X.F. Jin, X.M. Jiang, F.Q. Liu, H.J. Qian, K. Sun, L.M. Wang, G. Rossi, Z.Q. Qiu, J. Shi, Phys. Rev. Lett., 94 (2005) 137210-137211.

[4] S.R. Dunsiger, J.P. Carlo, T. Goko, G. Nieuwenhuys, T. Prokscha, A. Suter, E. Morenzoni, D. Chiba, Y. Nishitani, T. Tanikawa, F. Matsukura, H. Ohno, J. Ohe, S. Maekawa, Y.J. Uemura, Nat. Mater., 9 (2010) 299-303.

[5] F. Wang, D.H. Lee, Science, 332 (2011) 200-204.

[6] T. Dietl, H. Ohno, F. Matsukura, J. Cibert, D. Ferrand, Science, 287 (2000) 10191022. 
[7] C. Zener, J. Met., 7 (1955) 619-630.

[8] K.J. Tauer, R.J. Weiss, Phys. Rev., 100 (1955) 1223-1224.

[9] G. Inden, in: Proc. CALPHAD V, Max Planck Institut fuer Eisenforschung, Duesseldorf, Germany, 1976, pp. 1-13.

[10] M. Hillert, M. Jarl, CALPHAD, 2 (1978) 227-238.

[11] S. Hertzman, B. Sundman, CALPHAD, 6 (1982) 67-80.

[12] R.J. Weiss, K.J. Tauer, A metallurgical side rule for determining magnetic properties of $3 \mathrm{~d}$ transition alloys, in: Theory of Alloy Phases, Cleveland, Ohio, 1956, pp. 290-300.

[13] Y.Y. Chuang, R. Schmid, Y.A. Chang, Metall. Trans. A, 16 (1985) 153-165.

[14] W. Xiong, H. Zhang, L. Vitos, M. Selleby, Acta Mater., 59 (2011) 521-530.

[15] W. Xiong, K. Grönhagen, J. Ågren, M. Selleby, J. Odqvist, Q. Chen, Solid State Phenom., 172-174 (2011) 1060-1065.

[16] W. Xiong, M. Selleby, Q. Chen, J. Odqvist, Y. Du, Crit. Rev. Solid State Mater. Sci., 35 (2010) 125-152.

[17] W. Xiong, J. Odqvist, P. Hedström, M. Selleby, M. Thuvander, Q. Chen, CALPHAD, 35 (2011) 355-366.

[18] O. Redlich, A.T. Kister, Indust. Eng. Chem., 40 (1948) 345-348.

[19] G. Inden, Scand. J. Metall., 20 (1991) 112-120.

[20] R.A. Reck, Phys. Rev. B, 9 (1974) 2381.

[21] D. Marko, T. Strache, K. Lenz, J. Fassbender, R. Kaltofen, Appl. Phys. Lett., 96 (2010).

[22] I. Ohnuma, H. Enoki, O. Ikeda, R. Kainuma, H. Ohtani, B. Sundman, K. Ishida, 
Acta Mater., 50 (2002) 379-393.

[23] I.H. Jung, S.A. Decterov, A.D. Pelton, H.M. Kim, Y.B. Kang, Acta Mater., 52 (2004) 507-519.

[24] B. Sundman, I. Ohnuma, N. Dupin, U.R. Kattner, S.G. Fries, Acta Mater., 57 (2009) 2896-2908.

[25] P. James, O. Eriksson, B. Johansson, I.A. Abrikosov, Phys. Rev. B, 59 (1999) 419430.

[26] J.O. Andersson, Int. J. Thermophys., 6 (1985) 411-419.

[27] A.T. Dinsdale, CALPHAD, 15 (1991) 317-425.

[28] Q. Chen, B. Sundman, J. Phase Equilib., 22 (2001) 631-644.

[29] G.A. Perez Alcazar, E. Galvao da Silva, J. Phys. F: Met. Phys., 17 (1987) 2323.

[30] E.P. Yelsukov, E.V. Voronina, V.A. Barinov, J. Magn. Magn. Mater., 115 (1992) 271-280.

[31] M. Fallot, Ann. Phys., 6 (1936) 305-387.

[32] M. Shiga, T. Kikawa, K. Sumiyama, Y. Nakamura, J. Magn. Soc. Jpn., 9 (1985) 187-190.

[33] F. Stein, M. Palm, Int. J. Mater. Res., 98 (2007) 580-588.

[34] D.J. Chakrabati, P.A. Beck, J. Phys. Chem. Solids, 32 (1971) 1609-1615.

[35] D. Parsons, W. Sucksmith, J.E. Thompson, Philos. Mag., 3 (1958) 1174-1184.

[36] M.J. Besnus, A. Herr, A.J.P. Meyer, J Phys F Met Phys, 5 (1975) 2138-2147.

[37] A.T. Aldred, B.D. Rainford, J.S. Kouvel, T.J. Hicks, Phys. Rev. B, 14 (1976) 228234.

[38] M.V. Nevitt, A.T. Aldred, J. Appl. Phys., 34 (1963) 463-468. 
[39] C.G. Shull, M.K. Wilkinson, Phys. Rev., 97 (1955) 304.

[40] Y. Liang, C.P. Guo, C.R. Li, Z.M. Du, J. Alloys Compd., 460 (2008) 314-319.

[41] J.O. Andersson, B. Sundman, CALPHAD, 11 (1987) 83-92.

[42] C.-P. Chin, S. Hertzman, B. Sundman, TRITA-MAC-0203, KTH, (1987).

[43] J. Cieslak, M. Reissner, W. Steiner, S.M. Dubiel, Phys. Status Solidi A, 205 (2008) 1794-1799.

[44] D.A. Read, E.H. Thomas, J. Phys. Chem. Solids, 29 (1968) 1569-1572.

[45] A.F. Guillermet, Z. Metallkd., 78 (1987) 700-709.

[46] M. Hillert, J. Alloys Compd., 320 (2001) 161-176.

[47] I. Ohnuma, T. Gendo, R. Kainuma, G. Inden, K. Ishida, ISIJ Int., 49 (2009) 12121219.

[48] L. Vinokurova, A. Vlaso, V. Ivanov, M. Pardavi-Horvath, E. Schwab, in: V.G. Veselago, L.I. Vinokurova (Eds.) Proceedings of the institute of general physics academy and the sciences of the USSSR: The magnetic and electron structures of transition metals and alloys, Nova Science Publishers, Commack, 1988, pp. 1-43.

[49] L. Kaufman, J. Phase Equilib. Diffus., 30 (2009) 418-428.

[50] T. Kemeny, B. Fogarassy, S. Arajs, C.A. Moyer, B Am Phys Soc, 22 (1977) 309310. 


\section{Figure captions}

Fig. 1. Different types of (a) magnetic moment and (b) magnetic entropy in the Fe-Ni system.

Fig. 2. Illustration of describing magnetic phase diagram of a hypothetical A-B system by using the AFM factor, the left column sub-figures (a) and (c) are the case with bec structure, while the right ones (b) and (d) are with non-bcc structure.

Fig. 3. Magnetic phase diagrams of a hypothetical A-B system with the bcc structure. Symbols stand for the imaginary experimental data.

Fig. 4. The upper figure (a) is the magnetic phase diagram of a hypothetical A-B system, while the lower figure (b) is the diagram of magnetic moment at $0 \mathrm{~K}$, in which the short dashed line is the mean magnetic moment, long dashed line is the artificial mean magnetic moment resulted by using the AFM factor, and the solid line is the effective magnetic moment.

Fig. 5 Illustration of different magnetic phase diagrams described using the improved magnetic model. The curves indicating the negative absolute temperatures are plotted as the dashed lines.

Fig. 6 (Online color) Hypothetical 3D magnetic phase diagram for a ternary A-B-D system according to the improved magnetic model in this work.

Fig. 7. (Online color) Magnetic transition temperature for the $\mathrm{Al}-\mathrm{Fe}, \mathrm{Al}-\mathrm{Cr}$ and $\mathrm{Cr}-\mathrm{Fe}$ systems. Experimental data for the magnetic transition temperatures in the bcc structure are from the work in Refs. [29-34]. Red dashed lines are the calculated magnetic transition temperatures according to the previous assessments [24, 40, 41], while the blue ones represent the magnetic transition temperatures calculated in this work using the improved magnetic model. 
Fig. 8. (Online color) Magnetic moment for the Al-Cr-Fe bcc alloys at $0 \mathrm{~K}$. The experiment data are from the work in Refs.[30-32, 35-39] . Blue solid symbols denote the effective magnetic moment. Red open symbols indicate the mean magnetic moment. Black solid symbols are for the local spin magnetic moments of $\mathrm{Fe}$ atoms, while the shadowed symbols are for the local spin magnetic moments of $\mathrm{Cr}$ atoms which show the value added by 2.0. Black solid and dashed lines are used to guide the eyes. Red dashed lines are the calculated mean magnetic moment according to the previous assessments [24, 40, 41], while the blue lines represent the effective magnetic moment calculated in this work using the improved magnetic model.

Fig. 9. (Online color) (a) Calculated magnetic ordering energy according to the simplified magnetic model implemented in current standard CALPHAD approach. (b) Calculated magnetic ordering energy according to the improved magnetic model in this work. (c) The difference between magnetic ordering energies calculated from the models before and after modification. (d) The three dimensional view of the results in (c).

Fig. 10. (Online color) A possible magnetic phase diagram of an A-B system for a certain structure. The red chained line is the Curie temperature. Green and blue shaded parts compose the FM region.

Fig. 11. Magnetic phase diagram of the Fe-Rh ordered bcc alloys according to the work by Vinokurova et al.[48] Phase transition temperatures were determined from different measurements according to magnetization, susceptibility and residual magnetization. Dashed lines are used for guiding the eyes. 


\section{Highlights}

- The standard magnetic model in the state-of-the-art CALPHAD approach is improved.

- The concept of effective magnetic moment is introduced as a measure of maximum magnetic entropy.

- The low temperature CALPHAD method calls for meticulous investigation of the magnetic phase diagrams.

- A case study on the Al-Cr-Fe system demonstrates the importance of careful consideration of magnetic contribution to the Gibbs energy. 
Improved magnetic model for Low temperature CALPHAD

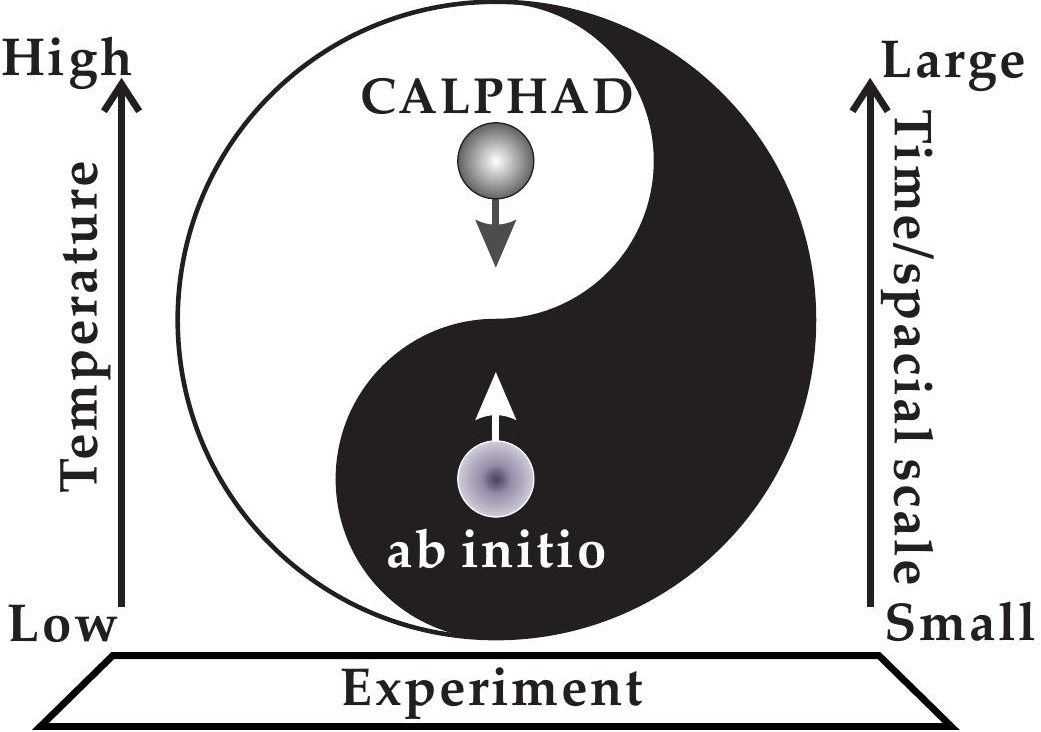

Case study










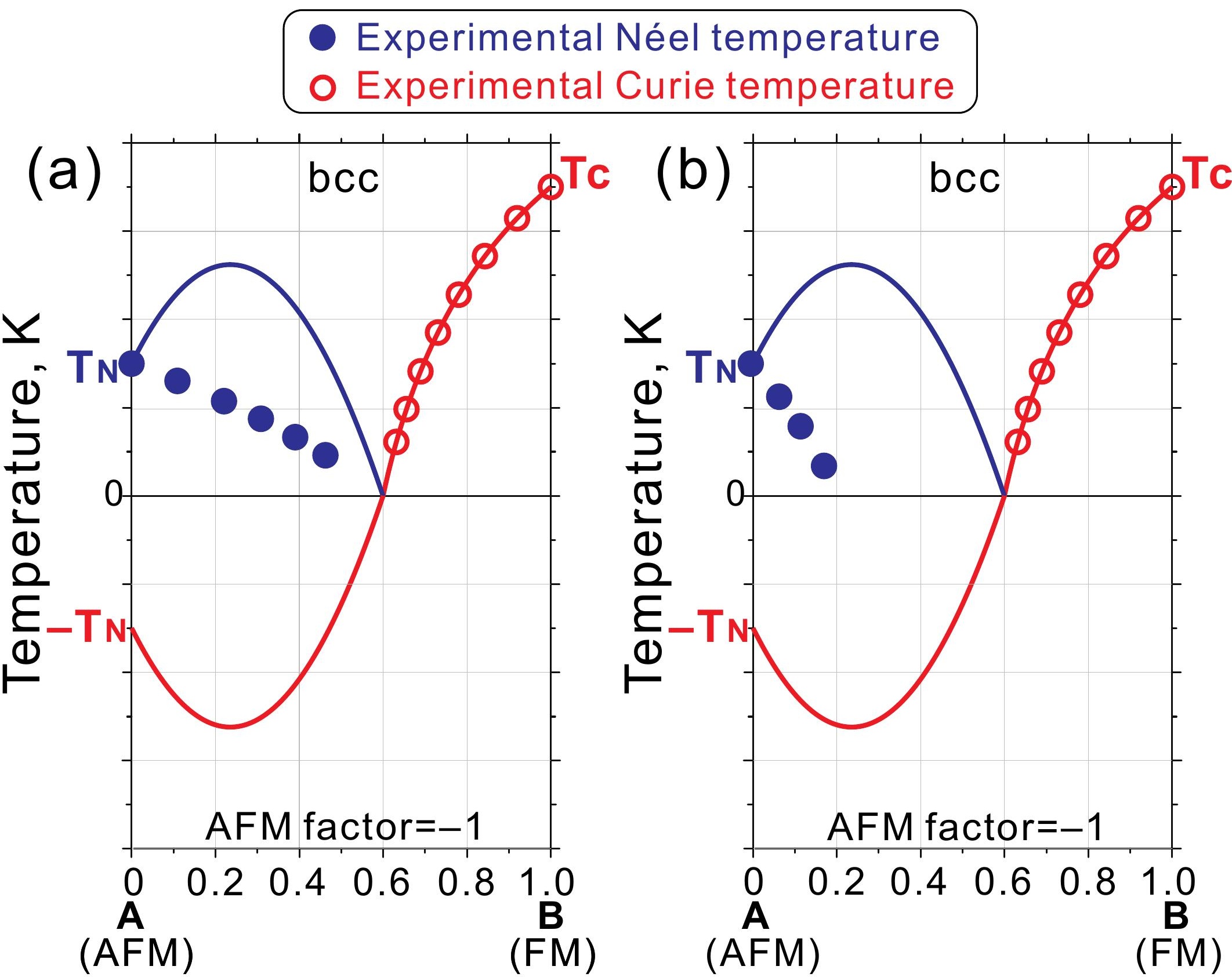


Figure 6

(a)

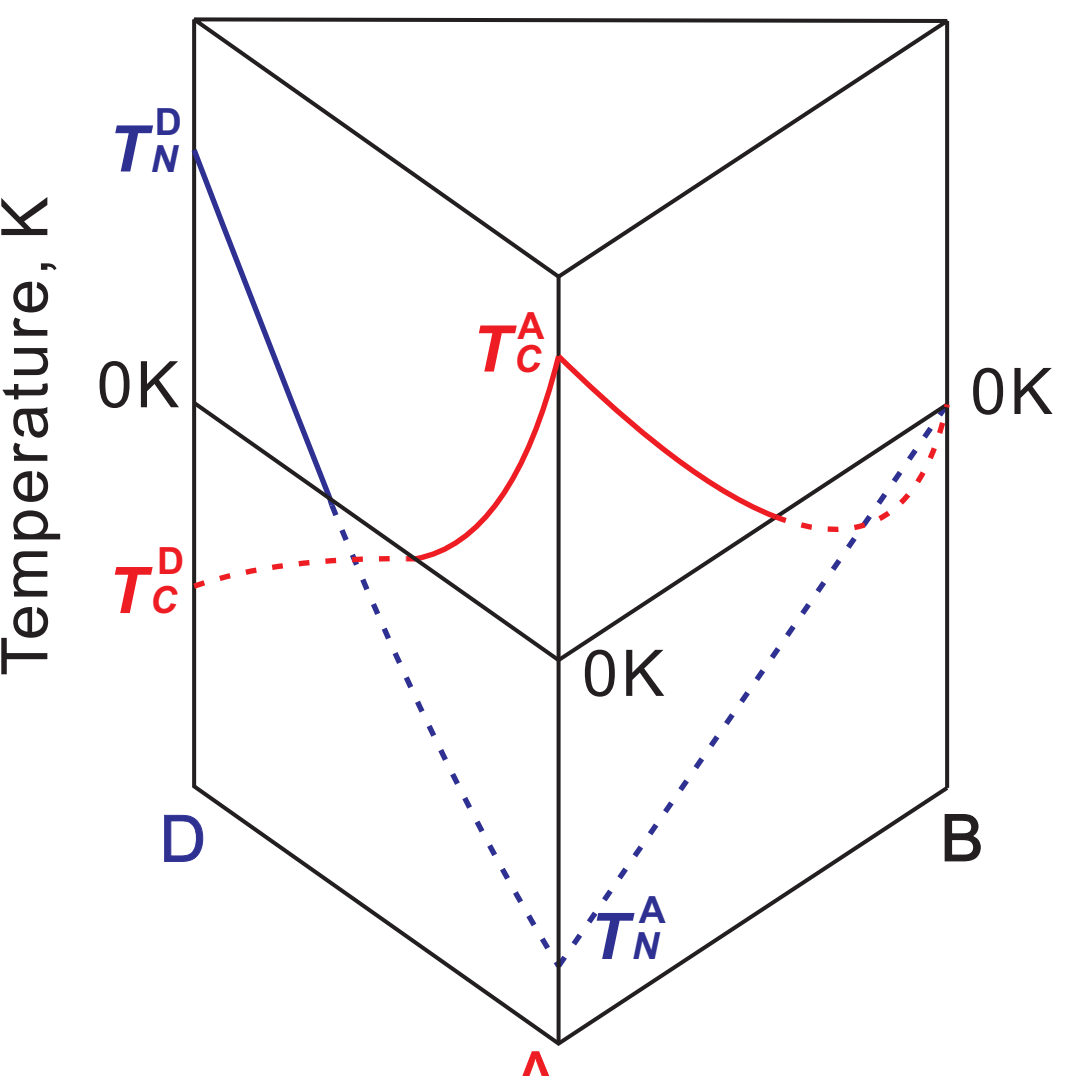

(b)

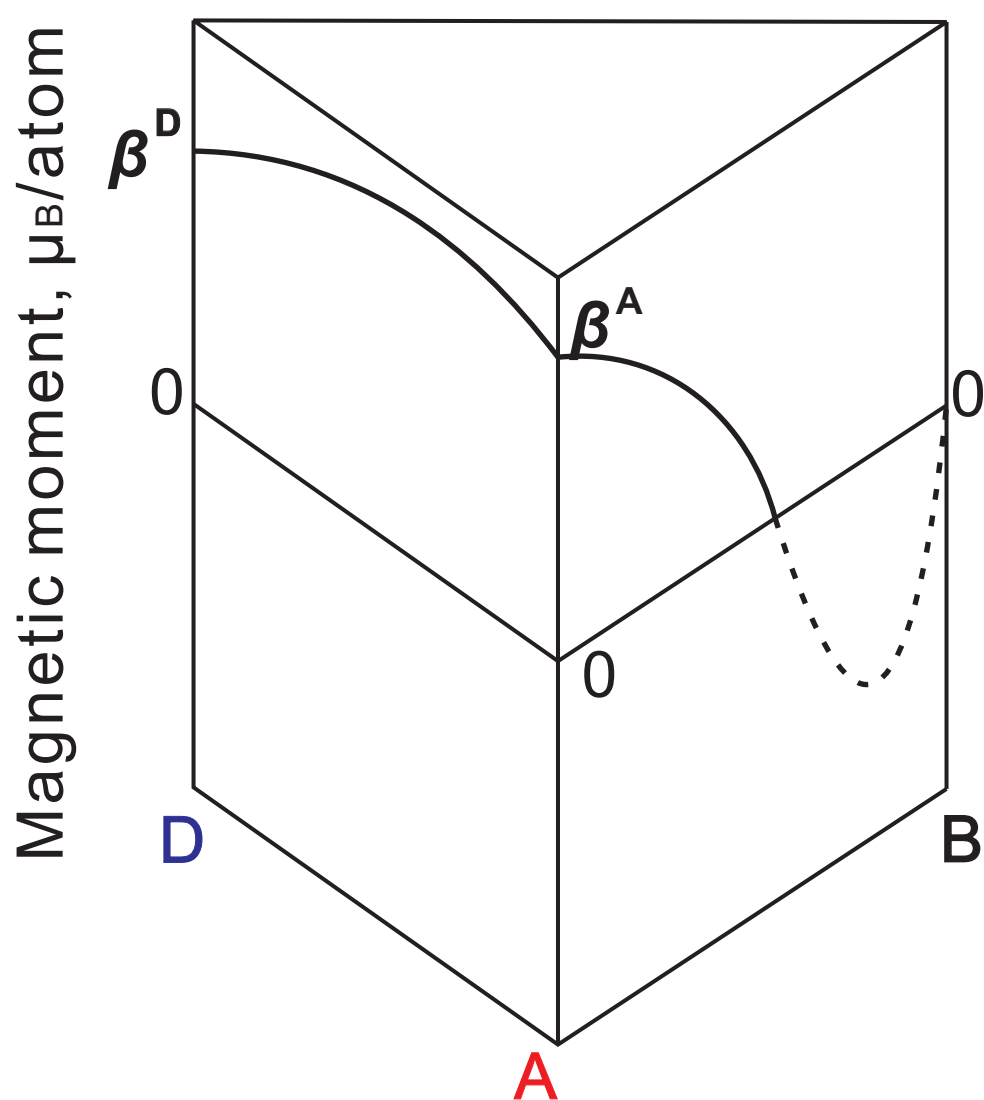




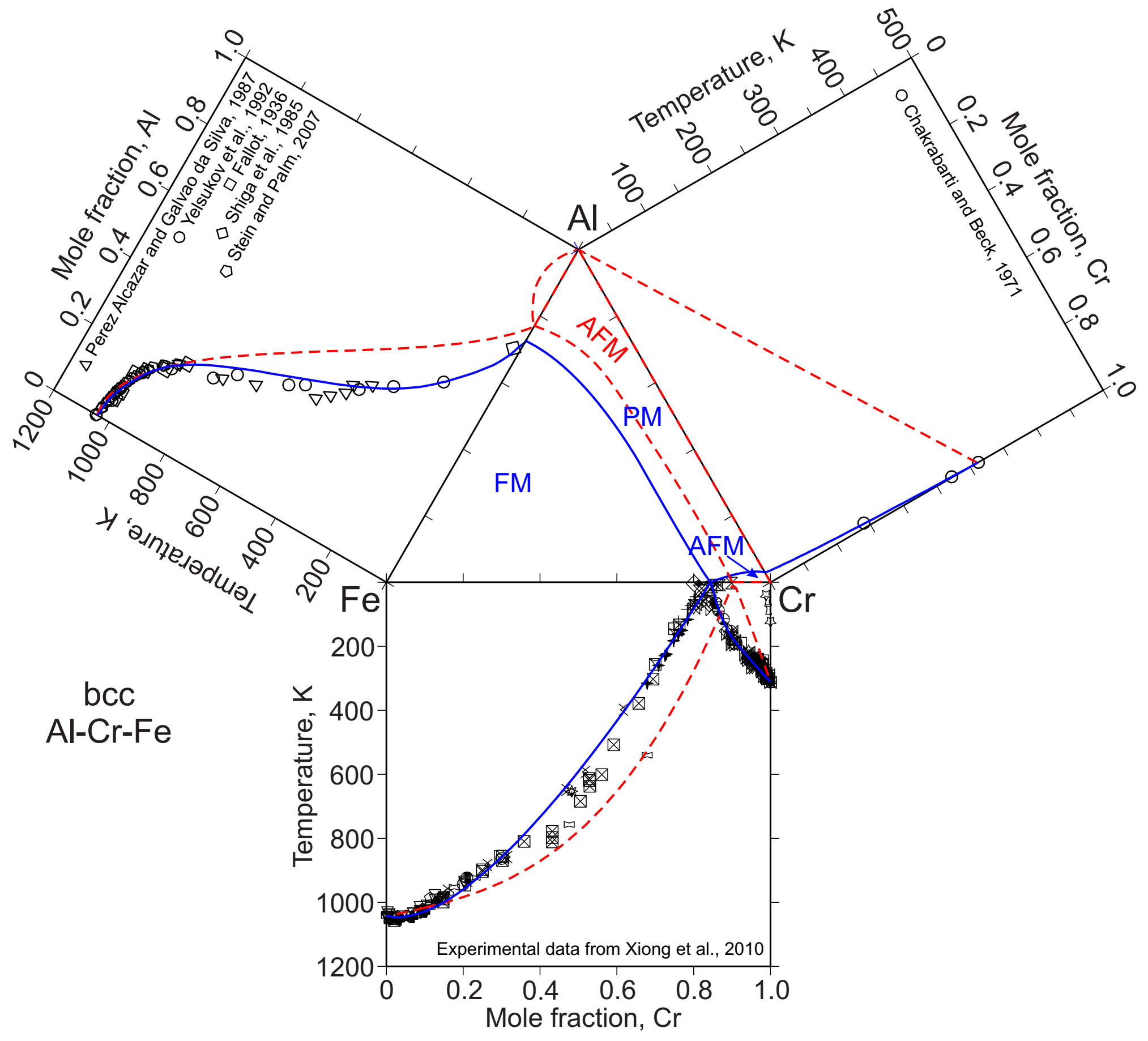




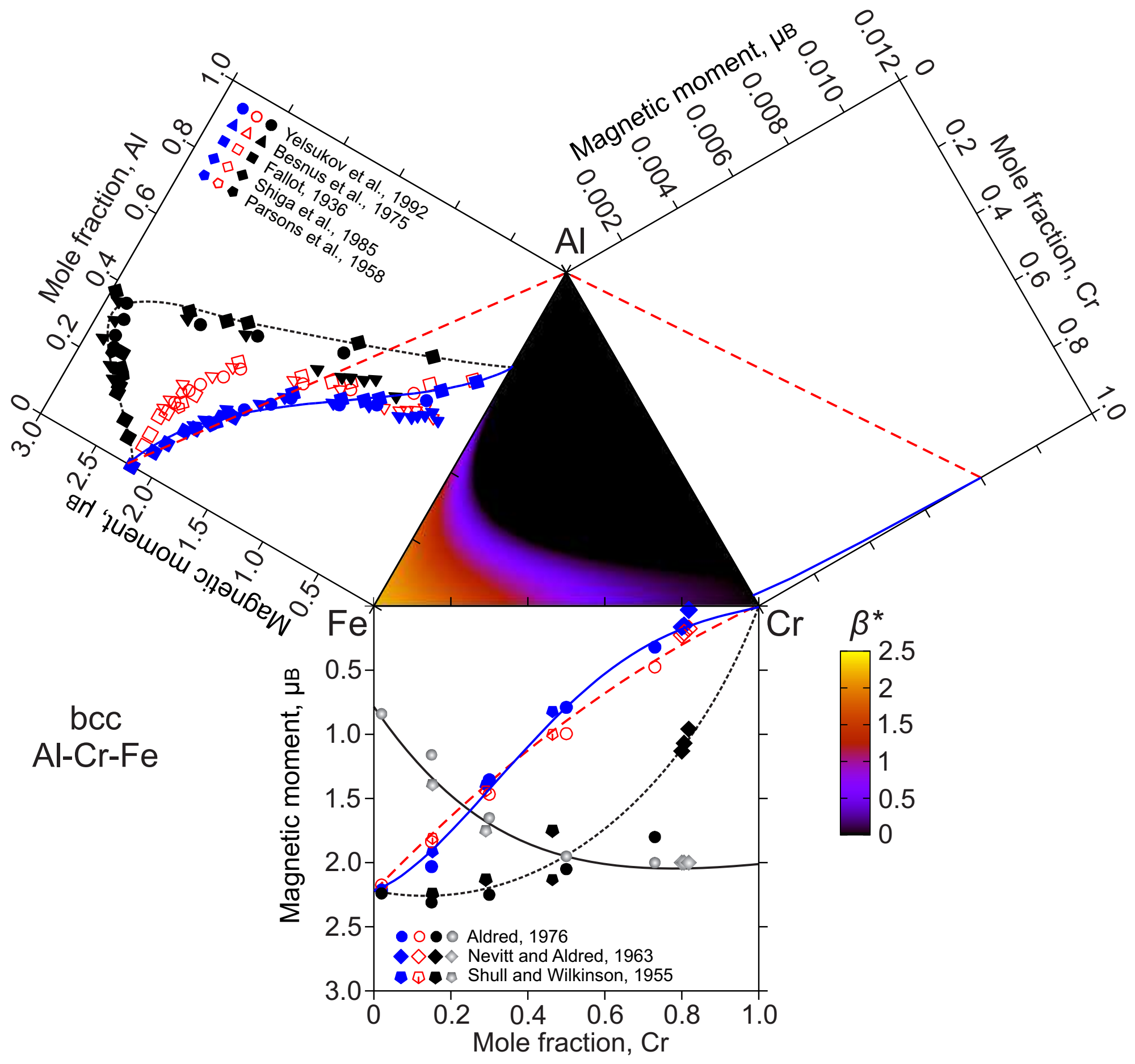


(a) Simplified IHJ model



(b) Model adopted in this work

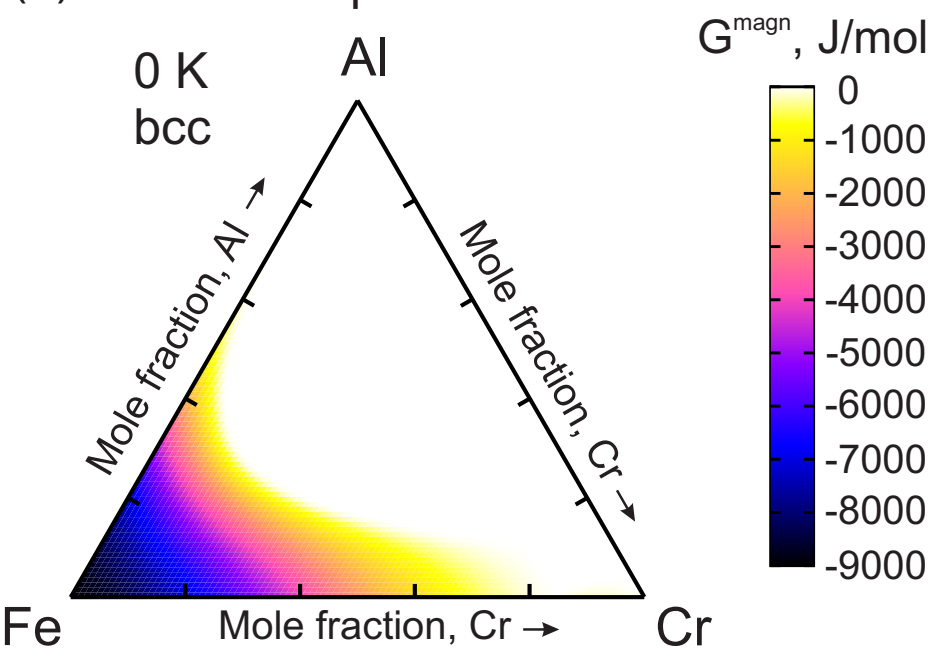

(c) Difference of Magnetic Ordering Energy between these two models



(d)






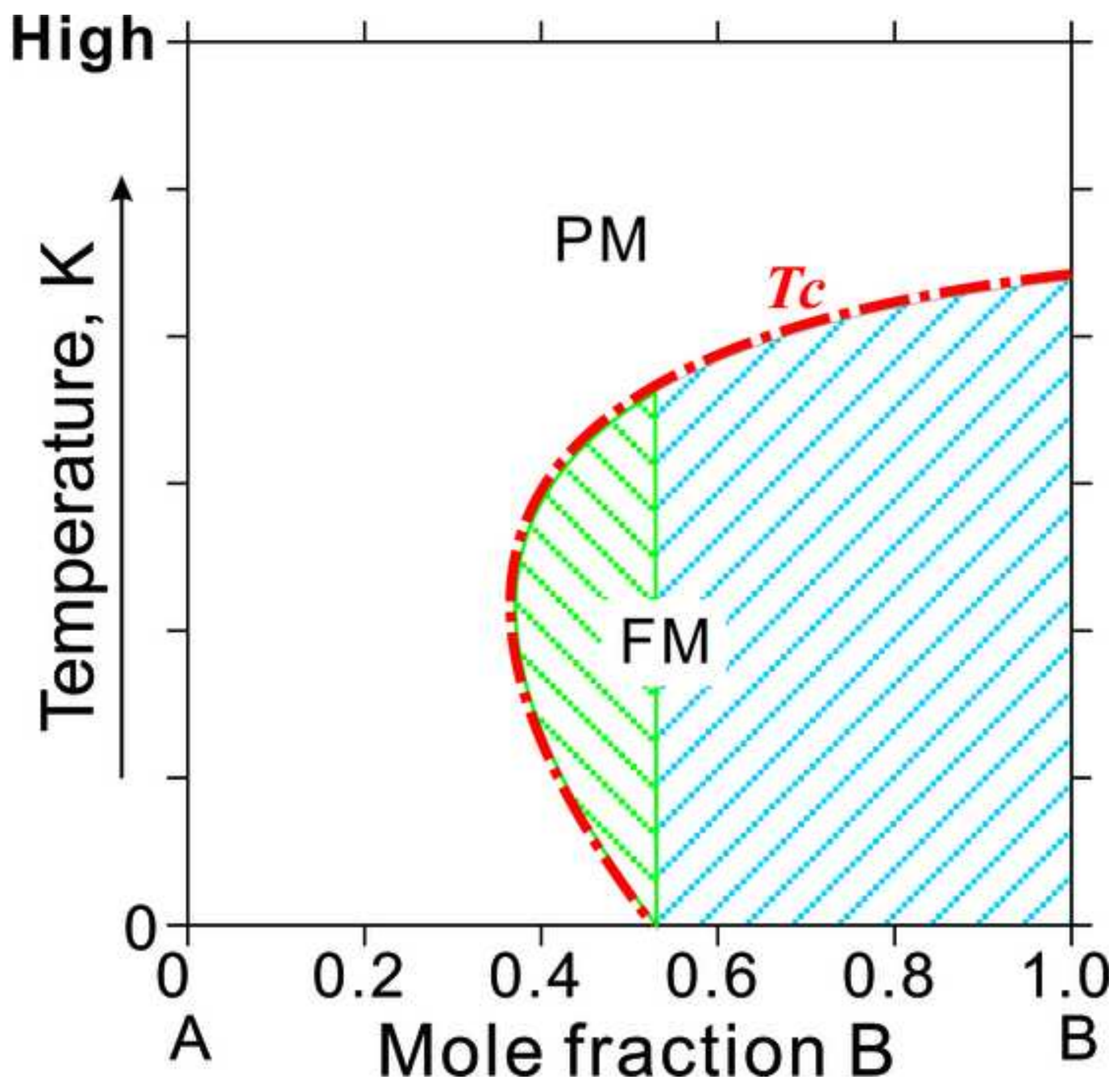




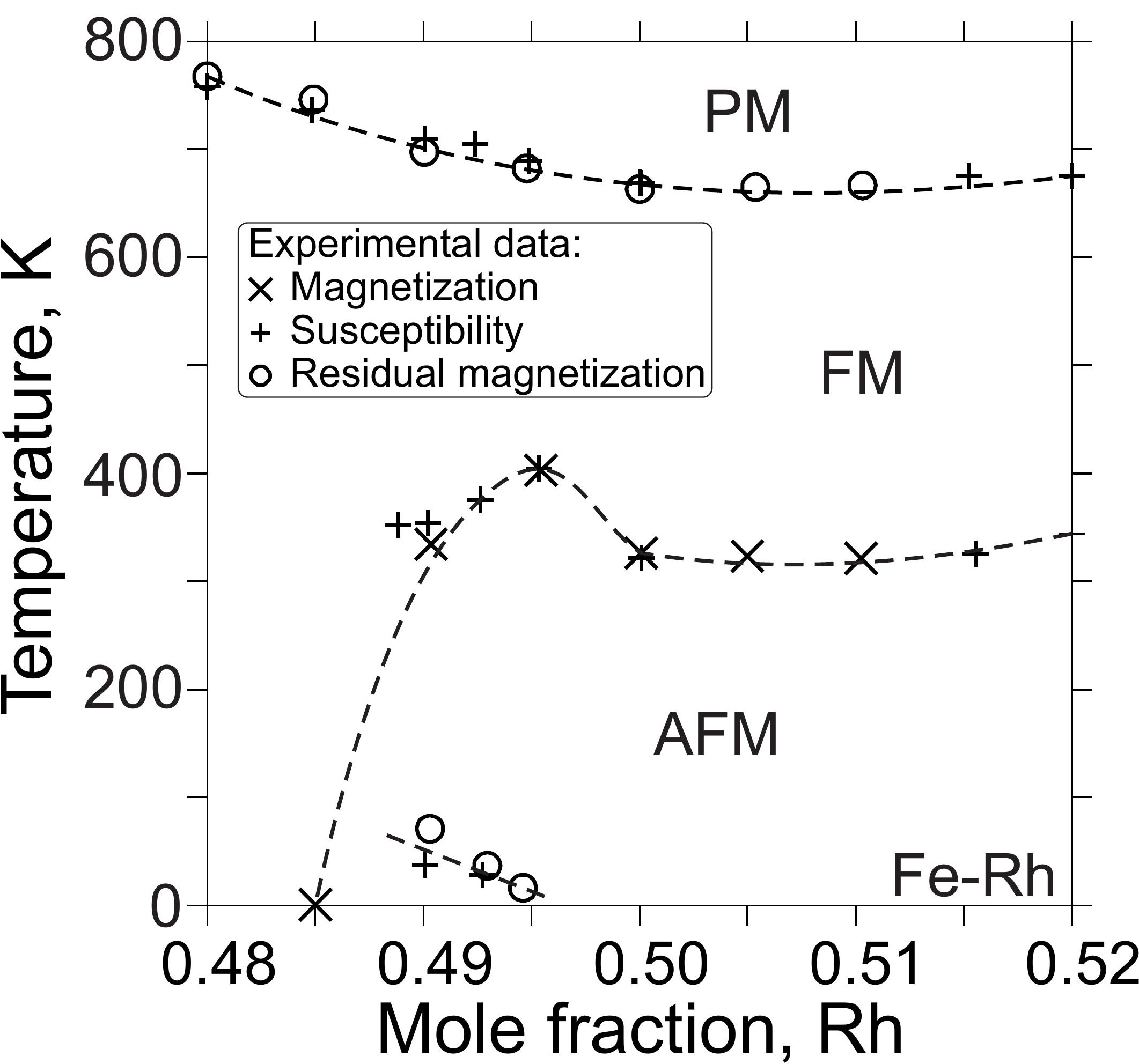


